\title{
Chiral symmetry breaking in fundamental and sextet fermion representations of SU(3) color*
}

\author{
Zoltán Fodor \\ Department of Physics, University of Wuppertal \\ Gau $\beta$ strasse 20, D-42119, Germany \\ Jülich Supercomputing Center, Forschungszentrum \\ Jülich, D-52425 Jülich, Germany \\ Email: fodorebodri.elte.hu

\section{Kieran Holland} \\ Department of Physics, University of the Pacific \\ 3601 Pacific Ave, Stockton CA 95211, USA \\ Email: khollandepacific.edu

\section{Julius Kuti} \\ Department of Physics 0319, University of California, San Diego \\ 9500 Gilman Drive, La Jolla, CA 92093, USA \\ E-mail: jkuti@ucsd.edu

\section{Dániel Nógrádi} \\ Institute for Theoretical Physics, Eötvös University \\ H-1117 Budapest, Hungary \\ Email: nogradiabodri. elte.hu
}

\section{Chris Schroeder}

Department of Physics, University of Wuppertal

Gaußstrasse 20, D-42119, Germany

E-mail: chris.schroeder@gmail.com

We report new results for lattice gauge theories with twelve fermion flavors in the fundamental representation and two fermion flavors in the two-index symmetric (sextet) representation of the $\mathrm{SU}(3)$ color gauge group. Both models are important in searching for a viable composite Higgs mechanism in the Beyond the Standard Model (BSM) paradigm. We subject both models to opposite hypotheses inside and outside of the conformal window. In the first hypothesis we test chiral symmetry breaking ( $\chi \mathrm{SB}$ ) with its Goldstone spectrum, $F_{\pi}$, the $\chi \mathrm{SB}$ condensate, and several composite hadron states as the fermion mass is varied in a limited range with our best effort to control finite volume effects. Supporting results for $\chi \mathrm{SB}$ from the running coupling based on the force between static sources is also presented. In the second test for the alternate hypothesis we probe conformal behavior driven by a single anomalous mass dimension under the assumption of unbroken chiral symmetry. Our results show very low level of confidence in the conformal scenario.

The XXVIII International Symposium on Lattice Field Theory, Lattice2010

June 14-19, 2010

Villasimius, Italy

\footnotetext{
*Based on talks at the conference by J. Kuti and Kieran Holland.
} 


\section{Introduction}

We report $\chi$ SB studies of two important gauge theories which attracted a great deal of attention in the lattice community and off-lattice as well. To establish the chiral properties of a gauge theory close to the conformal window is notoriously difficult. If the chiral symmetry is broken, the fundamental parameter $F$ of the chiral Lagrangian has to be small in lattice units $a$ to control cut-off effects. Since the chiral expansion has terms with powers of $N_{f} M_{\pi}^{2} / 16 \pi^{2} F^{2}$, reaching the chiral regime with large number of fermion flavors is particularly difficult. The range of $a M_{\pi}$ values where leading chiral logs can be identified unambigously will require simulations in very large volumes which is not in the scope of this study with twelve fermion flavors in the fundamental representation of the $S U(3)$ color gauge group. The sextet representation with two fermion flavors is considerably closer to the range of chiral perturbation theory in our simulations. Consistency requirements of chiral logs which fit the sextet results will require to get closer to the loop expansion of the continuum chiral Lagrangian, or the comprehensive application of staggered $S U(2)$ chiral perturbation theory on coarser lattices. We will make a case in this report that qualitatively different expectations inside and outside of the conformal window allow tests of the two mutually exclusive hypotheses without reaching down to the chiral logs at very small pion masses.

Below the conformal window chiral symmetry is broken at zero fermion mass with a gap in the composite hadron spectrum except the associated massless Goldstone multiplet. The analytic form of the chiral Lagrangian as a function of the fermion mass can be used to detect chiral log corrections, or to differentiate from conformal exponents in the transitional region before the chiral $\operatorname{logs}$ are reached at low enough Goldstone pion masses. Approximations to gauge theories with $\chi \mathrm{SB}$, like their effective Nambu-Jona-Lasinio description in the large $\mathrm{N}$ limit, are consistent with this analysis. In sharp contrast, the spectrum inside the conformal window is gapless in all channels in the chiral limit and the scale dependence of physical quantities is governed by the single critical exponent $\gamma$ which controls the fermion mass dependence of composite operators and their correlators.

The two competing hypotheses are tested for both gauge theories studied here in search for their chiral properties. There is a fundamental difference between the two hypotheses as implied by their respective spectra. $\chi \mathrm{SB}$ creates a fundamental scale $F$ in the theory separated from the composite hadron scale with its residual baryon gap in the chiral limit. The pion mass can be varied from the $\chi \mathrm{SB}$ scale $F$ to the hadron scale with a transition from the chiral log regime to a regime without chiral analysis. The conformal phase has no intrinsic scale. With $\chi \mathrm{SB}$ this is expected to lead to fermion mass dependence of the spectrum in the chiral log regime, or above it, quite different from the conformal behavior which is very tightly constrained near the chiral limit of the spectrum with a single critical exponent $\gamma$ in the absence of any intrinsic scale. In a regime where lattice cutoff effects are negligible, this difference should be sufficient for tests whether the chiral loop expansion is reached, or not, on the low $F$ scale.

In Section 2 we present new results for the gauge model with twelve fermions in the fundamental representation. A new kind of gauge dynamics is expected to appear at intermediate distances with walking gauge coupling, or a conformal fixed point. This remained controversial with recent efforts from five lattice groups $[1,2,3,4,5]$. We made considerable progress to resolve the controversies including tests of the chiral condensate and the spectrum which favor chiral symmetry 
breaking with unusual chiral dynamics. Applying the $\chi$ SB hypothesis to the Goldstone pion, $F_{\pi}$, the chiral condensate, and the stable nucleon state collectively leads to a result of $\chi^{2} / \mathrm{dof}=1.22$ representing high level of confidence. With the conformal hypothesis we find $\chi^{2} /$ dof $=8.79$ representing very low level of confidence. Applying global analysis to all states we measured, the contrasting behavior is somewhat less dramatic but remains significant. New results on the running coupling from the static force and our simulation of a rapid finite temperature transition in Polyakov loop distributions, reported elsewhere and expected in association with $\chi \mathrm{SB}$ and its restoration, provide further support for our findings.

In Section 3 we report new results for the two-index symmetric (sextet) color representation with two fermion flavors. The model holds promise for the composite Higgs mechanism in its simplest implementation without unwanted extra Goldstone bosons, or new electroweak multiplets. If $\chi \mathrm{SB}$ is found, the model is like the textbook introduction to Technicolor from QCD except that being close to the conformal window it becomes a viable BSM alternative without Electroweak precision problems. In a similar analysis introduced above, the sextet model was also subjected to the two mutually exclusive hypotheses. Applying the $\chi \mathrm{SB}$ hypothesis to the Goldstone pion, $F_{\pi}$, and the chiral condensate, a result of $\chi^{2} / \mathrm{dof}=1.24$ was found representing again high level of confidence. With the conformal hypothesis we find $\chi^{2} /$ dof $=6.96$ representing very low level of confidence.

We have used the tree-level Symanzik-improved gauge action for all simulations in this paper. The conventional $\beta=6 / g^{2}$ lattice gauge coupling is defined as the overall factor in front of the well-known terms of the Symanzik lattice action. Its value is $\beta=2.2$ for all simulations of the $N_{f}=$ 12 model and $\beta=3.2$ in the sextet model. The link variables in the staggered fermion matrix were exponentially smeared with two stout steps [6] and the precise definition of the action is given in [7]. The RHMC and HMC algorithms were deployed in all runs. Our error analysis of effective mass plots which combines systematic and statistical effects follows the frequentist histogram approach of the Budapest-Marseille-Wuppertal collaboration [8] in all simulations. The topological charge was monitored in the simulations with frequent changes observed over a considerable range.

\section{Twelve fermions in the fundamental $\mathrm{SU}(3)$ color representation}

The chiral Lagrangian for the Goldstone spectrum separated from the massive composite scale of hadrons exhibits, order by order, the well-known analytic form of powers in the fermion mass $m$ with non-analytic chiral log corrections generated from pion loops close enough to the chiral limit. The exact functions $F_{\pi}(m)$ and $M_{\pi}(m)$ will be approximated by an analytic form in powers of $m$ which is expected to hold over a limited $m$ range when the Goldstone pion is in transition from the chiral log regime closer to the composite hadron scale. Although this procedure has some inherent uncertainty before the chiral logs are reached in simulations, its sharp contrast with the non-analytic fermion mass dependence of the conformal hypothesis, governed by the single exponent $\gamma$, is sufficient to differentiate the two hypotheses.

First, we will illustrate the fitting procedure with results on the Goldstone spectrum, $F_{\pi}$, and the chiral condensate. This will be extended to the nucleon and some other composite hadron channels to probe parity degeneracy in the chiral limit. 


\subsection{Goldstone spectrum and $F_{\pi}$ from chiral symmetry breaking}
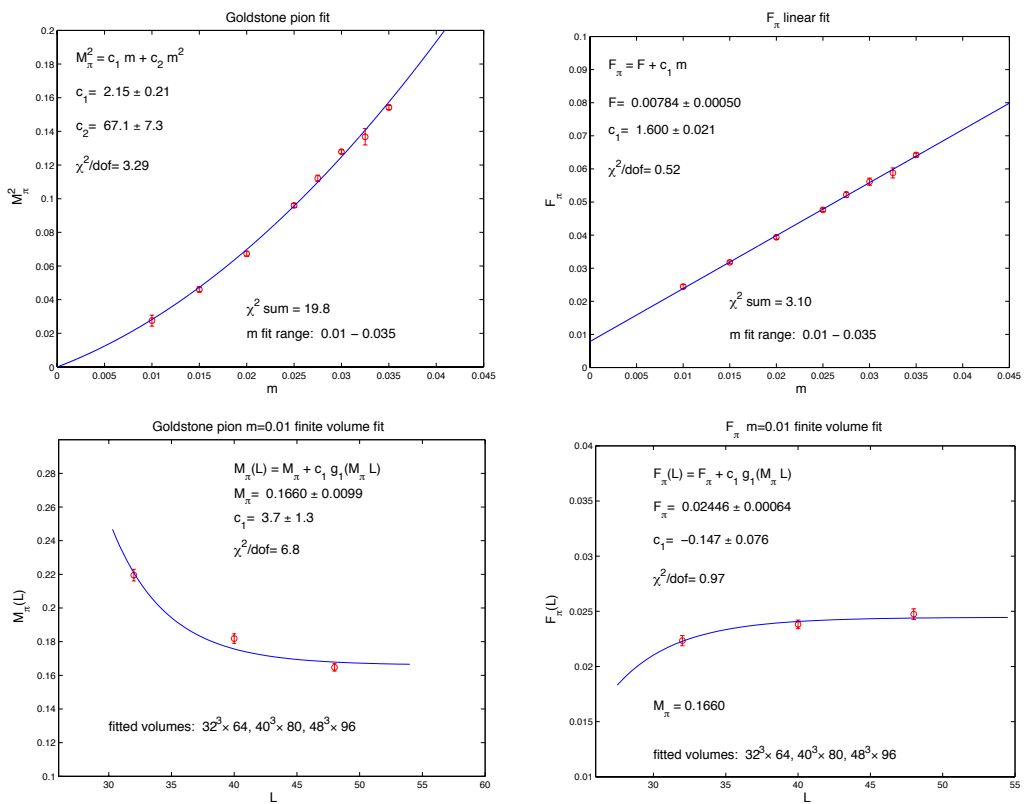

Figure 1: The Goldstone pion and $F_{\pi}$ from chiral symmetry breaking are shown with the fitting procedure described in the text. Representative finite volume fits are also shown. The infinite volume limit of $M_{\pi}$ was used in fits to $F_{\pi}$ and other composite hadron states, like the nucleon.

Figure 1 shows the Goldstone pion and $F_{\pi}$ as a function of the fermion mass $m$ in the range where we can reach the infinite volume limit with confidence. The power functions of the fitting procedure in $m$ contain the analytic contributions of the fourth order chiral Lagrangian to $M_{\pi}$ and $F_{\pi}$. Although we could fit the pion spectrum with the logarithmic term included, its significance remains unclear. The rapid variation of $F_{\pi}$ with $m$ clearly shows that we would need a dense set of data in the $m=0.003-0.01$ range to reach chiral logs at this gauge coupling. This requires lattice volumes well beyond the largest size $48^{3} \times 96$ which we could deploy in our simulations.

Efforts were made for extrapolations to the infinite volume limit. Finite volume scaling is very different under the hypotheses of the two different scenarios. At the lowest three $m$ values, for finite volume corrections to $M_{\pi}$ and $F_{\pi}$, and for all other states, we used the form

$$
\begin{aligned}
M_{\pi}\left(L_{s}, \eta\right) & =M_{\pi}\left[1+\frac{1}{2 N_{f}} \frac{M^{2}}{16 \pi^{2} F^{2}} \cdot \widetilde{g}_{1}(\lambda, \eta)\right], \\
F_{\pi}\left(L_{s}, \eta\right) & =F_{\pi}\left[1-\frac{N_{f}}{2} \frac{M^{2}}{16 \pi^{2} F^{2}} \cdot \widetilde{g}_{1}(\lambda, \eta)\right],
\end{aligned}
$$

where $\widetilde{g}_{1}(\lambda, \eta)$ describes the finite volume corrections with $\lambda=M_{\pi} \cdot L_{s}$ and aspect ratio $\eta=L_{t} / L_{s}$ from the lightest pion wrapping around the lattice and coupled to the measured state. The form of $\widetilde{g}_{1}(\lambda, \eta)$ is a complicated infinite sum which contains Bessel functions and requires numerical evaluation. Since we are not in the chiral log regime, the pre-factor of the $\widetilde{g}_{1}(\lambda, \eta)$ function was replaced by a fitted coefficient. The leading term of the function $\widetilde{g}_{1}(\lambda, \eta)$ is a special exponential Bessel function $K_{1}(\lambda)$ which dominates in the simulation range. The fitting procedure could be 
viewed as the approximate leading treatment of the pion which wraps around the finite volume, whether in chiral perturbation theory, or in Luscher's non-perturbative finite volume analysis. The $M_{\pi} L_{s}>4$ lore for volume independence is clearly not applicable in the model. We need $M_{\pi} L_{s}>8$ to reach volume independence. The infinite volume limits of $M_{\pi}$ and $F_{\pi}$ for each $m$ were determined self-consistently from the fitting procedure using Eqs. (2.1) based on a set of $L_{s}$ values with representative fit results shown in Figure 1. In the higher $m$ range finite volume effects were hard to detect and even for the lowest $m$ values sometimes volume dependence was not detectable for the largest lattice sizes.

Non-Goldstone pion spectra, quite different from those found in QCD, are shown in Figure 2 using standard notation. They are not used in our global analysis. The three states we designate as i5Pion, ijPion and scPion do not show any noticeable taste breaking or residual mass in the $m \rightarrow 0$ chiral limit. The scPion is degenerate with the i5Pion and both are somewhat split from the true Goldstone pion. The ijPion state is further split as expected but the overall taste breaking is very small across four pion states. This is a fairly strong indication that the coupling constant $\beta=2.2$ where all runs are performed is close to the continuum limit. A very small residual mass at $m=0$ is not excluded for some non-Goldstone states depending on the details of the fitting procedure.
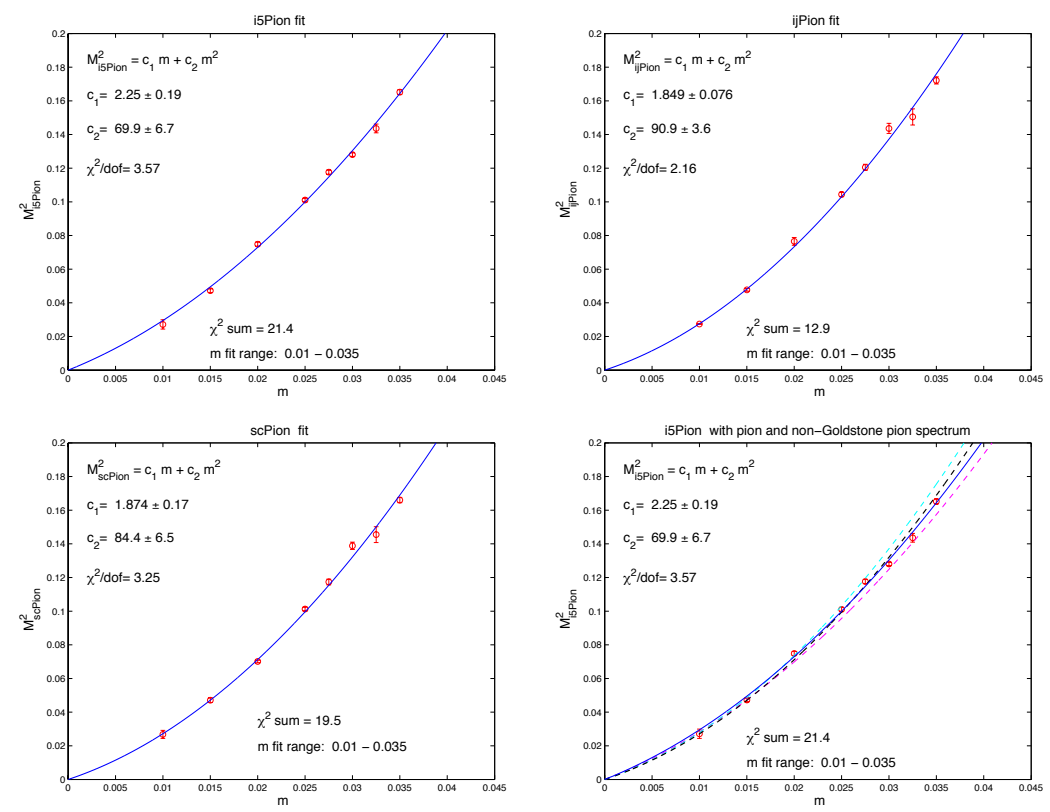

Figure 2: The non-Goldstone pion spectrum is shown. The composite lower right plot redisplays the i5Pion data together with fits to the Goldstone pion (magenta), i5Pion (solid blue), scPion (black), and ijPion (cyan).

\subsection{Chiral condensate}

The chiral condensate $\langle\bar{\psi} \psi\rangle$ summed over all flavors is dominated by the linear term in $m$ from UV contributions. The quadratic (or linear) fit in Figure 3 gives a small non-vanishing condensate in the chiral limit which is in the expected ballpark from the GMOR relation $\langle\bar{\psi} \psi\rangle=12 F^{2} B$ with the measured low $F$ and $B$ of the order one. The deficit between the two sides of the GMOR relation is sensitive to the fitting procedure and the determination of $B$. Adding a quadratic term to the fit is 

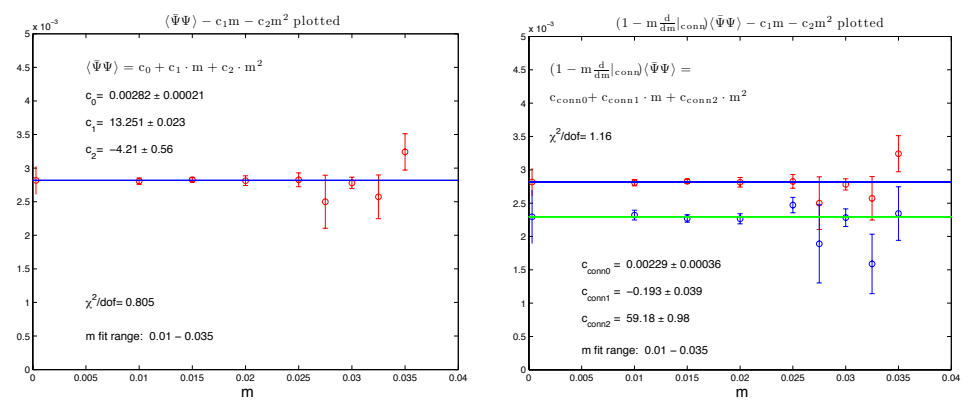

Figure 3: The chiral condensate and its subtracted derivative version (both have to converge to the same chiral limit) are shown after the removal of the non-constant part of respective fit functions of the form $c_{0}+c_{1} m+c_{2} m^{2}$. The left side shows the fit to $\langle\bar{\psi} \psi\rangle$ condensate data after the removal of the fitted $c_{1} m+c_{2} m^{2}$ part with fit error on the chiral limit value of $c_{0}$ at $m=0$. The right side superimposes on the left side plot the subtracted derivative condensate fit (blue points) (with fit coefficients displayed) after the removal of the $c_{1} m+c_{2} m^{2}$ part. The error of $c_{0}$ at $m=0$ for this quantity is also shown and consistent with the direct determination of the chiral limit from $\langle\bar{\psi} \psi\rangle$. For any given $m$ always the largest volume condensate data is used since the finite volume analysis is not complete. The relative sensitivity of the analysis to the lowest two or three $m$ values can be eliminated by extended systematics .

a small effect and trying to identify chiral logs is beyond the scope of our simulation range. For an independent determination, we also studied the subtracted chiral condensate operator

$$
\left(1-\left.m \frac{d}{d m}\right|_{c o n n}\right) \cdot\langle\bar{\psi} \psi\rangle
$$

which is determined from zero momentum connected correlators. The removal of the derivative term significantly reduces the dominant linear part of the $\langle\bar{\psi} \psi\rangle$ condensate. We find it reassuring that the two independent determinations give consistent non-vanishing results in the chiral limit as clearly shown in Figure 3.

It should be noted that the $M_{\pi}$ values in the fitting range of $m$ in our analysis are below the fitting range of previous $N_{f}=12$ work on the chiral condensate work with considerably more uncertainty from using the higher range [3]. In all fits we were on a fine-grained lattice in the pion mass range $a M_{\pi}=0.16-0.39$ and rho mass range $M_{\rho}=0.2-0.47$. In contrast, the previous study [3] which reported conformal behavior was in the $a M_{\pi}=0.35-0.67$ range and rho mass range $M_{\rho}=0.39-0.77$. Although our new results should be made even more definitive with higher accuracy and better control on the systematics, the evidence is quite suggestive for a small non-vanishing chiral condensate in the chiral limit.

\subsection{Composite hadron spectrum in the chiral limit}

It is important to investigate the chiral limit of other composite hadron states. They further test the gaps of physical states as the fermion mass $m$ is varied and the measured hadron masses are subjected to chiral analysis in the $m \rightarrow 0$ limit. Hadron masses also provide useful information on parity splits in several channels. One composite state of great interest is the Higgs particle, if there is a chiral condensate close to the conformal window. We will briefly review new results on the nucleon state with its parity partner, the isospin partner of the Higgs state, and the $\rho-A_{1}$ splitting.

The fermion mass dependence of the nucleon and its parity partner is shown in Figure 4 with finite volume analysis at one selected fermion mass $m=0.015$. The same finite volume fit is 

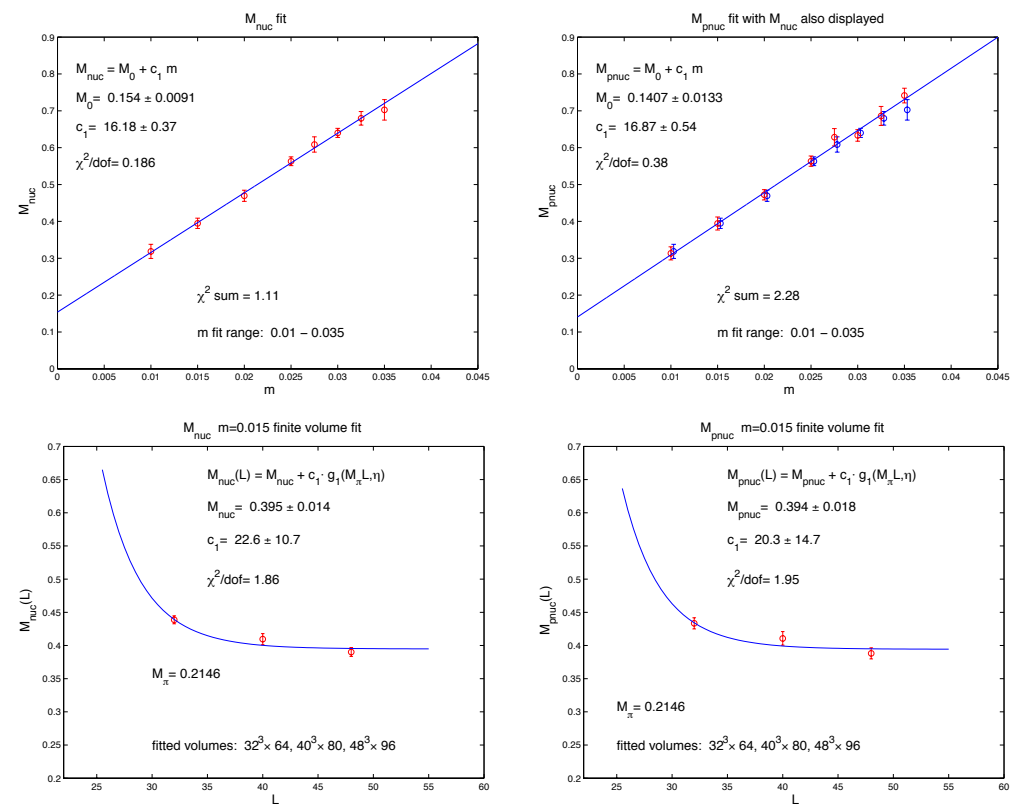

Figure 4: Nucleon and parity partner are fitted to the simplest linear form which is also the leading term of the chiral Lagrangian. The blue points on the right are the replotted nucleon data from the left to show the degeneracy of the two states. The lower two plots show finite volume fits.

applied as described earlier for the pion state. The leading chiral linear term in the fermion mass $m$ extrapolates to non-vanishing chiral limit. The parity partner is practically degenerate but this is not a surprise. Already with four flavors a near degeneracy was reported before by the Columbia group.

Figure 5 shows the fermion mass dependence of the Higgs particle without including the disconnected part of the relevant correlator. Strictly speaking, therefore, the state is the $f_{0}$ meson with non-zero isospin. Disconnected contributions in the correlator might shift the Higgs mass, an important issue left for future clarifications. Both the linear and the quadratic fits are shown together with the pseudo-goldstone scPion which is split down from the Higgs (that is, $f_{0}$ ) state. The two states would be degenerate in the chiral limit with unbroken symmetry. The Higgs state (as we call it) extrapolates to a nonvanishing mass in the chiral limit with $M_{H} / F$ ratio between 10 and 15 . This ratio is approximately 5 in the sextet model. Finally, Figure 6 shows the $\rho$-meson and its $A_{1}$ parity partner. Both states extrapolate to non-vanishing mass in the chiral limit. The split remains significant for all fermion masses and in the chiral limit.

\subsection{String tension and running coupling from the static force}

There are several ways to define a renormalized gauge coupling, for example, the Schrödinger Functional scheme or from square Wilson loops. We take the renormalized coupling as defined via the quark-antiquark potential $V(R)$, extracted from $R \times T$ Wilson loops where the time extent $T$ can be large. From the potential, one defines the force $F(R)$ and coupling $\alpha_{q q}(R)$ as

$$
F(R)=\frac{d V}{d R}=C_{F} \frac{\alpha_{q q}(R)}{R^{2}}, \quad \alpha_{q q}(R)=\frac{g_{q q}^{2}(R)}{4 \pi} .
$$



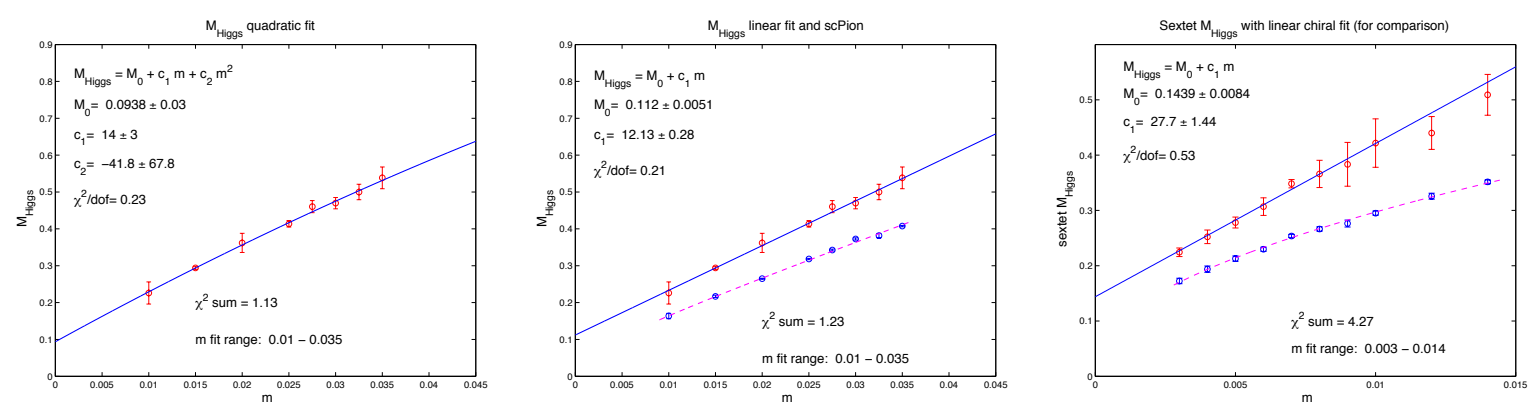

Figure 5: The $f_{0}$ state (we call it Higgs) and its splitting from the scPion state are shown. The linear fit in the middle plot works well for the Higgs $\left(f_{0}\right)$ state with little change when a quadratic term is included on the left. The blue scPion data points in the middle plot and the dashed magenta fit show the fit to the scPion state. The Higgs will became a resonance in the chiral limit, the missing disconnected part also contributing, so that Higgs predictions will be challenging in future work. For comparison the Higgs state is shown on the right from the sextet analysis with its blue scPion parity partner split with dashed magenta fit.
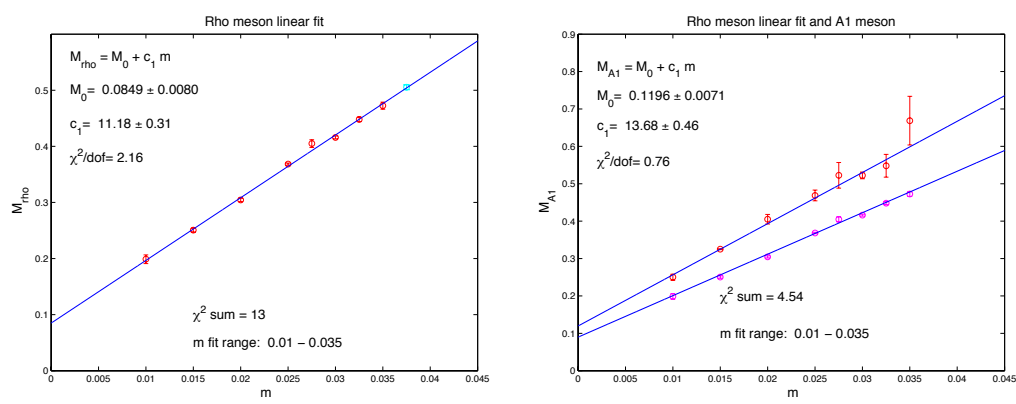

Figure 6: Rho meson and its splitting from the A1 meson are shown. On the right side the magenta points reproduce the data of the rho meson from the left together with its linear fit. The fit parameters on the right show the linear fit to the $A_{1}$ meson.

The coupling is defined at the scale $R$ of the quark-antiquark separation, in the infinite-volume limit $L \rightarrow \infty$. This is different from the scheme using square Wilson loops, where one has $\alpha_{W}(R, L)$ and one can choose finite $R$ with $L \rightarrow \infty$, or finite $L$ and fixed $R / L$ ratio. In the former case, these schemes are related via

$$
\alpha_{q q}(R)=\alpha_{W}(R)\left[1+0.31551 \alpha_{W}(R)+\mathscr{O}\left(\alpha_{W}(R)^{2}\right)\right]
$$

The $\beta$ function in the qq scheme is known to 3-loops. For $S U(3)$ gauge theory with $N_{f}=12$ fundamental flavors, the location of the infrared fixed point to 3-loop order is $\alpha_{q q}^{*}=0.3714 \ldots$ This is about $50 \%$ of the scheme-independent 2-loop value of $\alpha^{*}$, indicating that higher order corrections beyond 3-loop might not be negligible.

A range of lattice spacings, volumes and quark masses are studied in the running coupling project, we show results for the largest volume $48^{3} \times 96$ at $\beta=2.2$ and quark masses $m=0.01$ and 0.015 and for the $40^{3} \times 80$ run at $m=0.02$. To improve the measurement of $V(R)$, we use different levels of APE-smearing to produce a correlation matrix of Wilson loops, the lowest energy is extracted using the generalized eigenvalue method. We also improve the lattice force, which is naively discretized as $F(R+1 / 2)=V(R+1)-V(R)$. For the Symanzik gauge action, the 

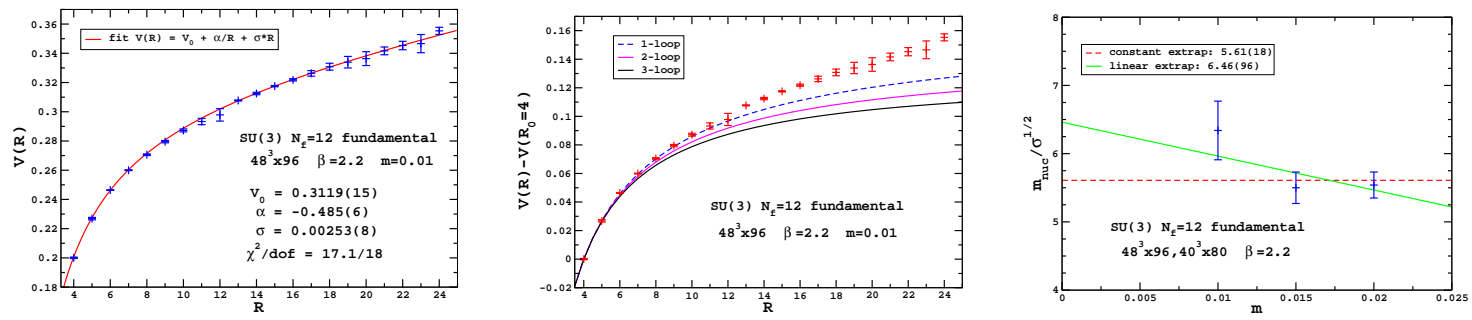

Figure 7: $V(R)$ data and fit for $m=0.01$ is plotted on the left and comparison with perturbation theory is plotted in the middle. The right side plot shows the string tension measured in nucleon mass units at $m=0.01,0.015,0.02$ and extrapolated to the chiral limit. The finite nucleon mass gap in the chiral limit implies finite string tension at $m=0$.

improvement is a relatively small effect, for example the naive value $R+1 / 2=4.5$ is shifted to $4.457866 \ldots$

In Figure 7 on the left we show the measured $V(R)$ fitted to the form

$$
V(R)=V_{0}+\frac{\alpha}{R}+\sigma R
$$

for $m=0.01$. The $m=0.015$ and $m=0.02$ runs are shown on the right of Figure 7. For all three masses, the resulting fits are good, with a clear signal of linear dependence and an effective string tension $\sigma$. The string tension decreases with the quark mass, its behavior in conjunction with the mass spectrum in the chiral limit is under investigation and the first result is shown in the figure. The finite nucleon mass gap in the chiral limit implies finite string tension at $m=0$.

The renormalized coupling $\alpha_{q q}(R)$ is a derivative of the potential $V(R)$ and hence more difficult to numerically measure via simulations. The most accurate comparison between lattice simulations and perturbation theory is directly of the potential $V(R)$ itself. This is naturally given by finite potential differences

$$
V(R)-V\left(R_{0}\right)=C_{F} \int_{R_{0}}^{R} \frac{\alpha_{q q}\left(R^{\prime}\right)}{R^{\prime 2}} d R^{\prime},
$$

where $R_{0}$ is some reference point where $\alpha_{q q}\left(R_{0}\right)$ is accurately measured in simulations. From this starting point, the renormalized coupling runs according to perturbation theory, at some loop order. The result is shown in the middle of Figure 7, with curves at 1-, 2- and 3-loop order for the potential difference. Although progress was made in studies of important finite volume effects, more work is needed to bring the systematics under full control. In the current state of the analysis the string tension and the fast running coupling are consistent with the $\chi S B$ hypothesis and do not support the conformal one.

\subsection{Testing the alternate hypothesis of conformal chiral symmetry}

The simulation results we presented for twelve fermions in the fundamental repesentation of the $S U(3)$ color gauge group favor the chiral symmetry breaking hypothesis. The pion state is consistent with a vanishing mass in the chiral limit and easy to fit with a simple quadratic function of the fermion mass. The non-Goldstone pion spectrum shows very little taste breaking at $\beta=2.2$ and the small splittings are consistent with expectations for staggered fermions with stout smearing. The $S O(4)$ degeneracies and splittings appear to follow the pattern of QCD although the fermion 

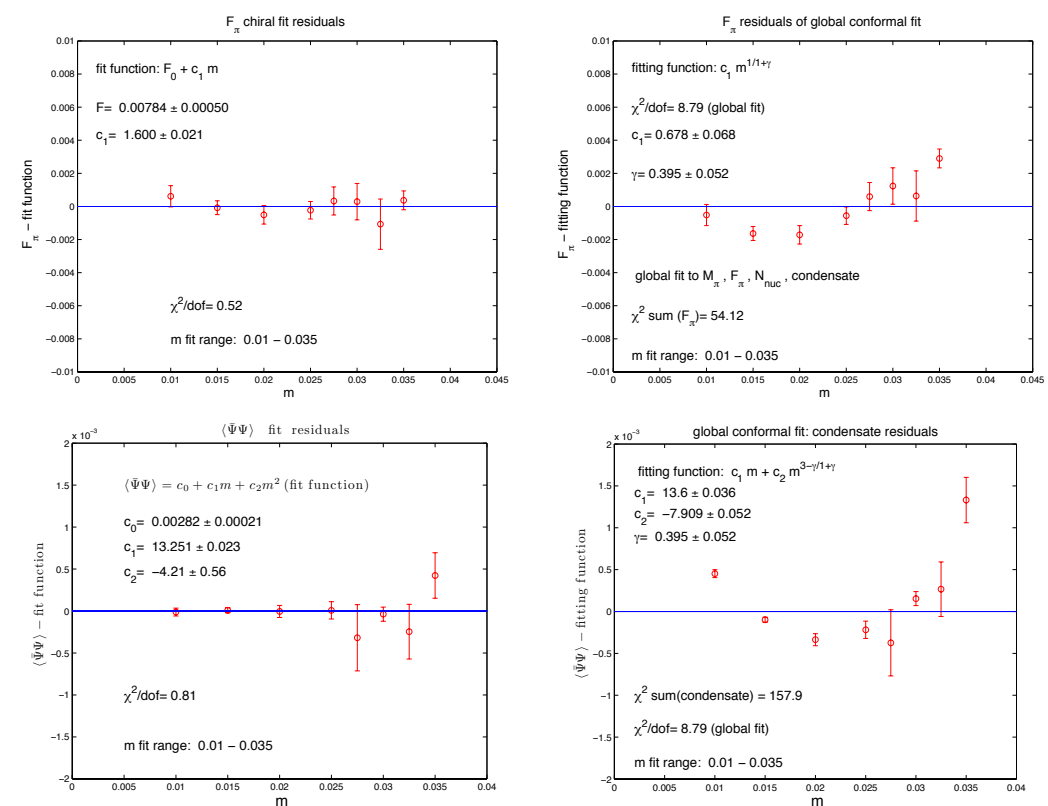

Figure 8: The $N_{f}=12$ chiral and conformal simultaneous fits in four channels are displayed for comparison in two select cases.

mass dependence is significantly different. The fundamental scale-setting parameter $F$ of chiral symmetry breaking is finite in the chiral limit. A non-vanishing chiral condensate is found in the chiral limit which is in the expected ballpark of the GMOR relation as suggested by the small value of $F$. The subtracted chiral condensate, after the dominant linear UV-contribution is removed, also yields a consistent non-vanishing condensate in the chiral limit. The nucleon states, the Higgs $\left(f_{0}\right)$ meson, the $\rho$ meson and $A_{1}$ meson extrapolate to non-vanishing masses in the chiral limit and considerable splits of some of the parity partner states persist at very low fermion masses toward the chiral limit. There seems to be an effective string tension indicating confinement-like behavior below the string-breaking scale and the running coupling has not shown signs of a fixed point slowdown. In addition, there seems to be a rapid finite temperature transition whose nature is unclear but hardly favors a conformal bulk phase. Our results are consistent with results reported in [4] but disagree with the chiral analysis of [3] and do not support the infrared fixed point reported in [1].

But is it possible that we mislead ourselves with the $\chi \mathrm{SB}$ interpretation? Can we interpret the results as conformal chiral symmetry? To decide this question, a fairly stringent test is possible. With the conformal hypothesis the mass dependence of all physical states is controlled by the anomalous dimension $\gamma$ for small fermion masses [11]. Each hadron and $F_{\pi}$ should scale as $M_{\pi} \approx$ $m^{1 / y_{m}}$ and $F_{\pi} \approx m^{1 / y_{m}}$ for small $m$ where $y_{m}=1+\gamma$. For small enough $m$ the value of $\gamma$ should be interpreted as $\gamma^{*}$ at the infrared fixed point. The chiral condensate is expected to have the behavior $\langle\bar{\psi} \psi\rangle \approx c \cdot m+m^{\frac{3-\gamma}{1+\gamma}}$ when $m \rightarrow 0$. We selected various subsets of states for a combined fit with universal critical exponent $\gamma$. We also fitted all measured states combined. Applying the conformal hypothesis to the chiral condensate, to $F$, to the pion state, and to the stable nucleon state collectively leads to a results with a $\chi^{2}$ sum of $\chi^{2}=229$ for 26 degrees of freedom with 
$\chi^{2} /$ dof $=8.79$. This indicates very low level of confidence in the hypothesis. The $\chi$ SB hypothesis gives $\chi^{2} /$ dof $=1.22$ for the same set of states. This was the result quoted in Section 1 . The chiral and conformal fits for two of the four fitted states with the quoted global results is shown in Figure 8. Applying the global analysis to all states we measured, the contrasting behavior is less pronounced but still significant. The results disfavoring the conformal hypothesis are significant. More work is needed for higher accuracy and full control of the systematics.

\section{Two fermions in sextet representation of the $\mathrm{SU}(3)$ color gauge group}

Our findings in the sextet model are consistent with chiral symmetry breaking. The gauge coupling $\beta=3.2$ where we report new results is more coarse-grained than the $\beta=2.2$ set in the $N_{f}=12$ model. Simulations at weaker bare coupling are underway. The chiral and conformal fitting procedures are identical to those we described earlier in the $N_{f}=12$ fermion model.

\subsection{Sextet Goldstone spectrum and $F_{\pi}$ from chiral symmetry breaking}
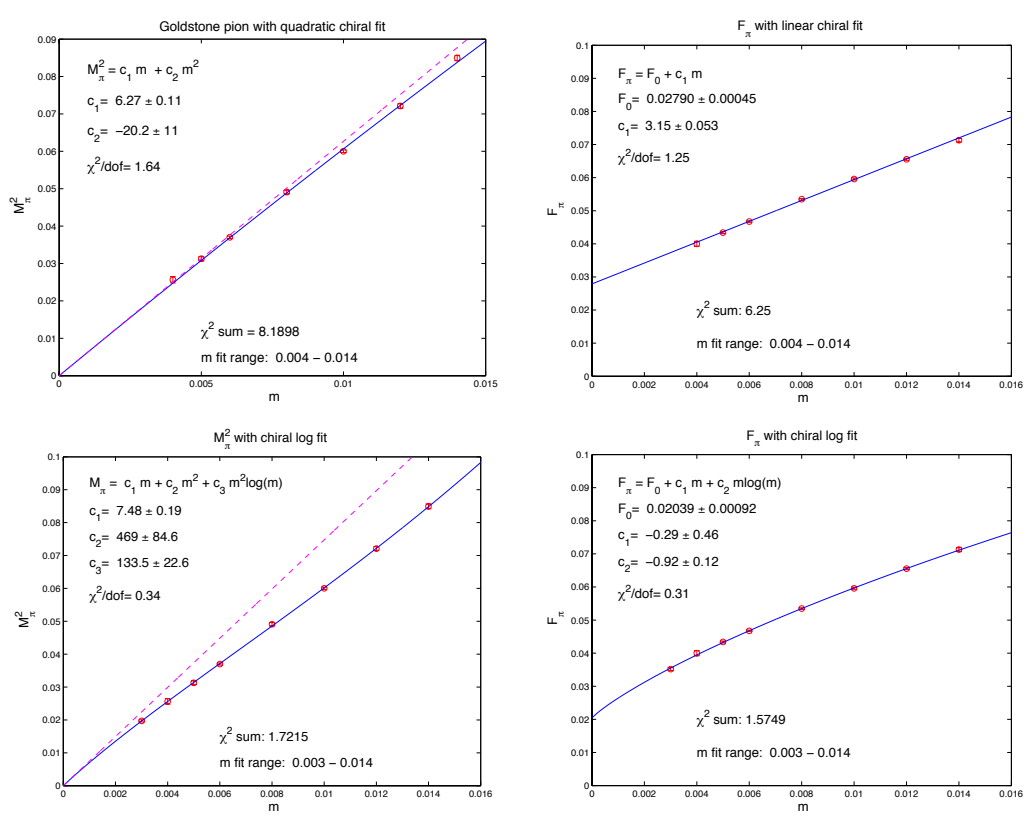

Figure 9: Analytic and logarithmic chiral fits for the Goldstone pion and $F_{\pi}$ are shown for $N_{f}=2$ sextet simulations with lattice size $32^{3} \times 64$ at $\beta=3.2$. The dashed lines show the linear part of the fits which estimate the $B$-parameter of the chiral Lagrangian. The upper two plots are analytic, the lower two plots include the leading chiral logs. The important role of the $m=0.003$ run is emphasized in the text.

Figure 9 shows the Goldstone pion and $F_{\pi}$ as a function of the fermion mass $m$ from $32^{3} \times 64$ lattices at the sextet gauge coupling $\beta=3.2$ with some finite volume control from $24^{3} \times 48$ runs. The upper two plots show analytic fits. At $m=0.004$ and higher the finite volume effects are small or negligible. Including the $m=0.003$ point in the analytic fit leads to a substantial increase in $\chi^{2}$. It remains unclear whether the $m=0.003$ run at $32^{3} \times 64$ lattice size is compatible with the infinite volume limit or favors an extrapolation. Including this point in the logarithmic chiral fit leads to an excellent result. It remains important to resolve the finite volume systematics at this fermion mass. 
Although we could fit $M_{\pi}$ and $F_{\pi}$ with the continuum chiral logarithm included, the separate sets of $F$ and $B$ from the fits are not quite self-consistent. A combined staggered $\mathrm{SU}(2)$ chiral perturbation theory fit is successful for simultaneous fits of $M_{\pi}$ and $F_{\pi}$ with the same pair of $F$ and $B$ values. The explicit cutoff dependent corrections to the $F$ and $B$ parameters would require further testing at weaker gauge coupling.

The sextet non-Goldstone pion spectrum is shown in Figure 10 using the same notation as earlier in the $N_{f}=12$ model. The three states we designate as i5Pion, ijPion, and scPion do show considerable taste breaking with residual mass in the $m \rightarrow 0$ chiral limit. The scPion remains degenerate with the i5Pion and they are split from the Goldstone pion. The ijPion state is further split from the i5Pion as expected. One plot at $\beta=3.25$ also shows that the taste breaking decreases at weaker gauge coupling. The non-Goldstone spectrum is more QCD-like than in the $N_{f}=12$ model.
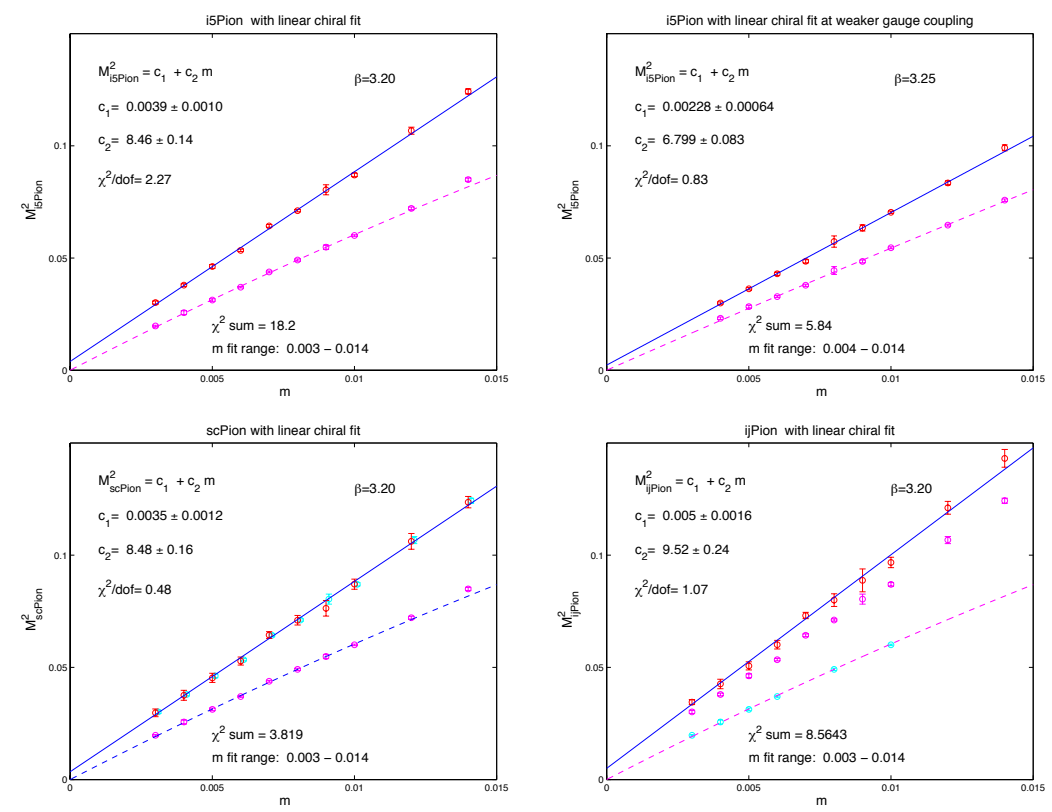

Figure 10: Sextet non-Goldstone spectrum is shown for three pion states. The upper left plot at $\beta=3.2$ shows the i5Pion with linear fit and non-zero intercept in the chiral limit. The magenta points show the Goldstone pion and its fit with considerable split from the i5Pion. The upper right plot is the same plot at $\beta=3.25$. The taste breaking is smaller at weaker gauge coupling. In the bottom left plot the red points with the linear fit shows the scPion. The cyan points show the i5Pion which remains degenerate with the scPion. The magenta points show the Goldstone pion. At the bottom right the red points and the linear fit show the ijPion with magenta points showing the i5Pion split downward. The cyan points with its fit show the Goldstone pion.

\subsection{Sextet chiral condensate}

The chiral condensate $\langle\bar{\psi} \psi\rangle$ summed over two flavors is dominated again by the linear term in $m$ from UV contributions. The quadratic (or linear) fit gives a small non-vanishing condensate in the chiral limit which is in the expected range from the GMOR relation $\langle\bar{\psi} \psi\rangle=2 F^{2} B$ with the fitted low $F$ value and $B$ estimated from the pion fit of figure 9. For an independent determination, we also studied the chiral condensate operator with the subtracted derivative terms as discussed 
earlier in the $N_{f}=12$ model. The fit to the condensate is shown in Figure 11 with non-vanishing chiral limit.
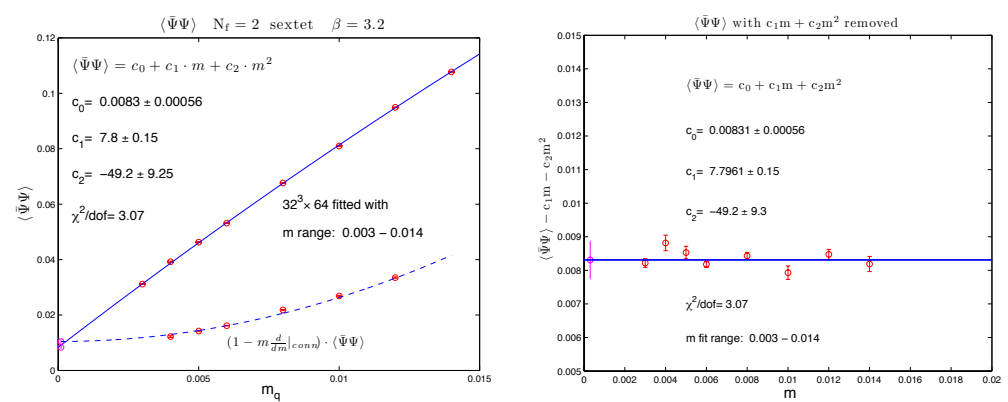

Figure 11: The sextet chiral condensate is shown on the left plot with fit and data points of the subtracted derivative. The right side shows the fit to $\langle\bar{\psi} \psi\rangle$ condensate data after the removal of the fitted $c_{1} m+c_{2} m^{2}$ part with fit error on the chiral limit value of $c_{0}$ at $m=0$.

\subsection{Testing the alternate hypothesis of conformal chiral symmetry in the sextet model}
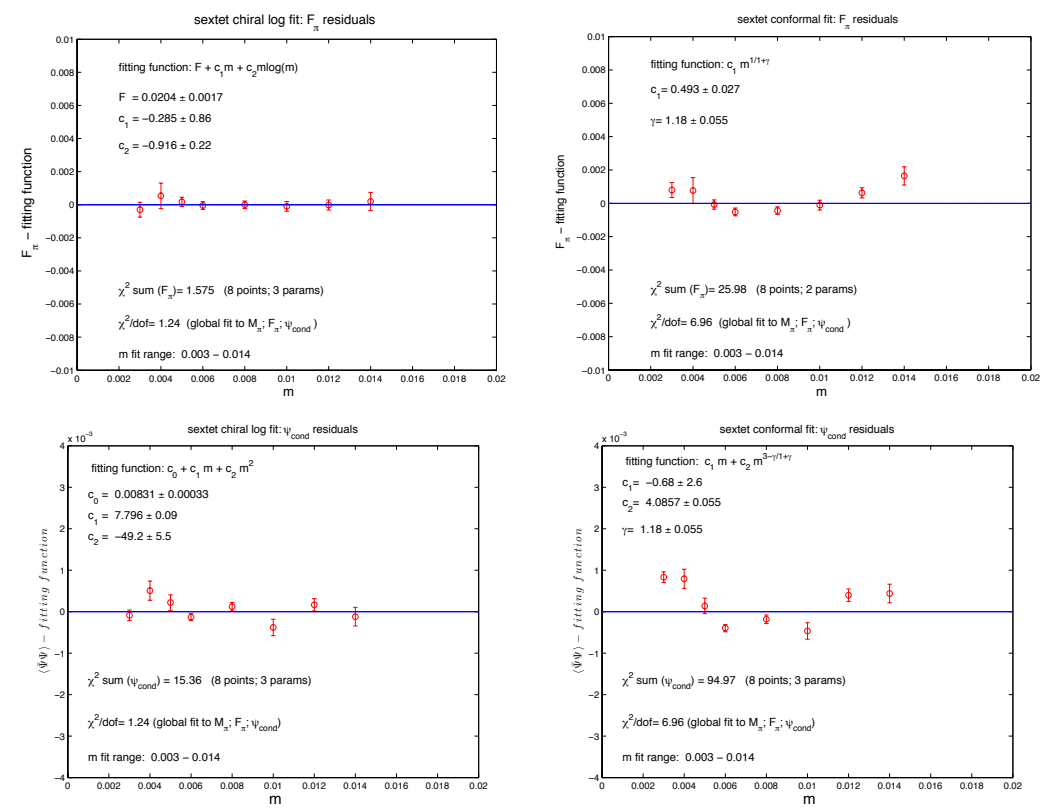

Figure 12: The sextet simultaneous fit in three channels.

All the sextet simulation results we presented favor the chiral symmetry breaking hypothesis. The pion state is consistent with a vanishing mass in the chiral limit and easy to fit with a simple quadratic function of the fermion mass. The fundamental controlling parameter $F$ of chiral symmetry breaking appears to be significantly non-vanishing in the chiral limit. The non-vanishing chiral condensate agrees reasonable well with the GMOR relation as calculated from the small fitted value of $F$ and $B$ estimated from the pion fit of Figure 9. The Higgs $\left(f_{0}\right)$ meson, the $\rho$ meson and $A_{1}$ meson extrapolate to non-vanishing masses. Applying the conformal hypothesis to $F_{\pi}, M_{\pi}$ and the $\langle\bar{\psi} \psi\rangle$ condensate, the combined fit gives $\chi^{2} /$ dof $=6.96$ in the $m=0.003-0.014$ fit range 
representing low level of confidence in the hypothesis. The combined chiral fit gives $\chi^{2} / \mathrm{dof}=1.24$ in the same range if the chiral $\log$ fits of Figure 9 are used for $M_{\pi}$ and $F_{\pi}$. Two comparison fits of this analysis are shown in Figure 12. We pointed out earlier that finite volume corrections in the $m=0.003$ run cannot be ruled out without further analysis. If we use the $m=0.004-0.014$ range in the conformal fits, we get a reduced $\chi^{2} /$ dof $=3.9$ value and the chiral fit is $\chi^{2} /$ dof $=1.29$. Further work is needed on the $m=0.003$ data.

In summary, our analysis of the sextet model favors the $\chi \mathrm{SB}$ hypothesis with considerable level of confidence and disagrees with earlier report from [12] using Wilson fermions in their sextet analysis. The results disfavoring the conformal hypothesis are not definitive but quite indicative. To sharpen the results even more continued work is needed for better control on the systematics at low fermion masses.

\section{Acknowledgments}

The simulations were performed using computational resources at Fermilab and JLab, under the auspices of USQCD and SciDAC, from the Teragrid structure and at Wuppertal. We are grateful to Kalman Szabo and Sandor Katz for their code development. This research is supported by the NSF under grants 0704171 and 0970137, by the DOE under grants DOE-FG03-97ER40546, DOEFG-02-97ER25308, by the DFG under grant FO 502/1 and by SFB-TR/55, and the EU Framework Programme 7 grant (FP7/2007-2013)/ERC No 208740. D. N. would like to thank the Aspen Center for Physics for invitation to the 2010 BSM summer program.

\section{References}

[1] T. Appelquist, G. T. Fleming and E. T. Neil, Phys. Rev. Lett. 100, 171607 (2008)

[2] Z. Fodor, K. Holland, J. Kuti, D. Nogradi and C. Schroeder, Phys. Lett. B 681, 353 (2009)

[3] A. Deuzeman, M. P. Lombardo and E. Pallante, Phys. Rev. D 82, 074503 (2010)

[4] X. -Y. Jin, R. D. Mawhinney, PoS LAT2009, 049 (2009).

[5] A. Hasenfratz, Phys. Rev. D82, 014506 (2010).

[6] C. Morningstar and M. J. Peardon, Phys. Rev. D 69, 054501 (2004)

[7] Y. Aoki, Z. Fodor, S. D. Katz, K. K. Szabo, JHEP 0601, 089 (2006).

[8] S. Durr, Z. Fodor, C. Hoelbling, S. D. Katz, S. Krieg, T. Kurth, L. Lellouch, T. Lippert et al., [arXiv:1011.2711 [hep-lat]].

[9] S. Catterall and F. Sannino, Phys. Rev. D 76, 034504 (2007)

[10] A. J. Hietanen, K. Rummukainen and K. Tuominen, Phys. Rev. D 80, 094504 (2009)

[11] L. Del Debbio, B. Lucini, A. Patella, C. Pica, A. Rago, Phys. Rev. D82, 014510 (2010).

[12] Y. Shamir, B. Svetitsky and T. DeGrand, Phys. Rev. D 78, 031502 (2008)

[13] J. B. Kogut, D. K. Sinclair, Phys. Rev. D81, 114507 (2010).

[14] E. Bilgici et al., Phys. Rev. D 80, 034507 (2009)

[15] N. Yamada, M. Hayakawa, K. -I. Ishikawa et al., PoS LAT2009, 066 (2009). 